FORMATION Formation emploi

Revue française de sciences sociales

96 | octobre-décembre 2006

Quand la qualification fait débat(s)

\title{
Les doctorants en sciences expérimentales : futurs collègues ou jeunes collègues?
}

Doctoral students in the experimental sciences: new colleagues or future

colleagues?

Doktoranden in den Exakten und Naturwissenschaften: Zukünftige oder junge

Kollegen?

Séverine Louvel

\section{OpenEdition}

Journals

Édition électronique

URL : http://journals.openedition.org/formationemploi/2543

DOI : 10.4000/formationemploi.2543

ISSN : 2107-0946

Éditeur

La Documentation française

Édition imprimée

Date de publication : 1 octobre 2006

Pagination : 53-66

ISSN : 0759-6340

Référence électronique

Séverine Louvel, «Les doctorants en sciences expérimentales : futurs collègues ou jeunes

collègues ? », Formation emploi [En ligne], 96 I octobre-décembre 2006, mis en ligne le 15 décembre

2009, consulté le 30 octobre 2020. URL : http://journals.openedition.org/formationemploi/2543; DOI : https://doi.org/10.4000/formationemploi.2543

(c) Tous droits réservés 


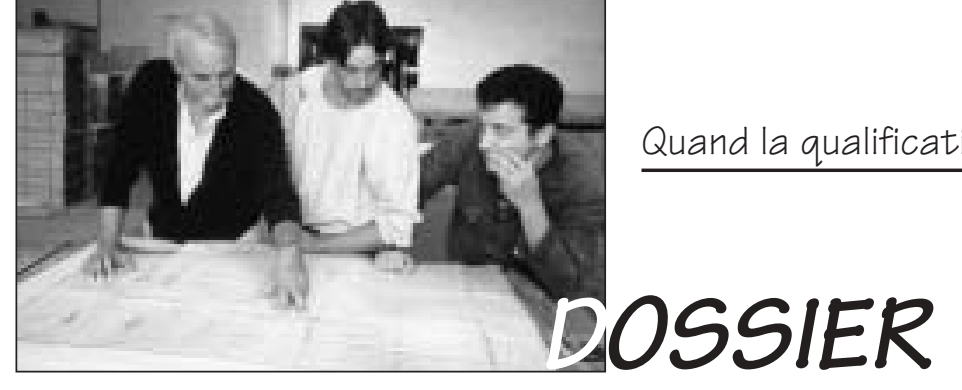

\section{Les doctorants en sciences expérimentales : futurs collègues ou jeunes collègues?}

Par Séverine Louvel*

Les doctorants occupent un double statut dans les laboratoires publics: ils se forment à la recherche et ils constituent une main-d'œuvre essentielle. Comment les doctorants et les autres membres des laboratoires reconnaissent-ils cette dualité socioprofessionnelle?

Les doctorants, qui représentent actuellement une proportion importante des personnels employés dans la recherche publique française ( $c f$. encadré $\mathbf{1})$, ont un double statut socioprofessionnel. Inscrits à l'université pour préparer un titre universitaire, ce sont des étudiants. Réalisant un projet de recherche et produisant des connaissances scientifiques et/ou technologiques, ils exercent aussi une activité de recherche sous la responsabilité d'un directeur de thèse.

Ce statut socioprofessionnel général recouvre des situations économiques et juridiques très contrastées. Deux tiers des doctorants n'ont pas de financement spécifique pour leur thèse: cette situation est courante en sciences humaines et sociales ${ }^{2}$. Le

\footnotetext{
${ }^{1}$ Merci à Catherine Marry, à Jean Saglio et aux lecteurs anonymes de la revue Formation Emploi pour leurs commentaires sur les versions antérieures de ce texte.

$282 \%$ des doctorants en sciences humaines et $30 \%$ des doctorants en sciences de la société ne sont pas financés pour leur thèse (DGRT, 2001).
}

dernier tiers est embauché pour une durée déterminée, généralement de trois ans, pour faire sa thèse ( $c f$. encadré 1 et tableau 1). Cette situation prévaut dans les sciences expérimentales, notamment en

* Séverine Louvel est sociologue, post-doctorante au laboratoire PACTE: Politiques publiques, action publique, territoires (CNRS, Grenoble Universités). Ses recherches portent sur l'emploi scientifique public et sur l'organisation des laboratoires de recherche dans le secteur public. Elle a notamment publié sur ces thèmes : "Mobilité et gestion des carrières dans la recherche chronique d'un échec annoncé ? » Gérer et comprendre, vol. 78, décembre 2004 ; La construction locale des laboratoires. Approche ethnographique de dynamiques d'évolution de laboratoires académiques en France. Thèse de sociologie, université Pierre Mendès France de Grenoble, 2005. 
chimie et en sciences de la vie ${ }^{3}$. La situation juridique des doctorants embauchés pour une durée déterminée a été clarifiée récemment. Elle stipule que leur rémunération n'est pas une bourse d'étude, mais la contrepartie d'un contrat de travail ${ }^{4}$.

Plusieurs analystes français (voir Futuris, 2005) soulèvent un paradoxe. Même les doctorants salariés pendant leur thèse parviennent plus facilement à faire reconnaître leur thèse (dans des cercles privés ou professionnels) comme une prolongation de leurs études que comme une première expérience de travail dans la

$380 \%$ des doctorants en chimie et sciences des matériaux et en biologie, médecine, santé ont un financement pour leur thèse (DGRT, 2001).

${ }^{4}$ La Direction des Affaires juridiques du ministère de l'Éducation nationale, de la Recherche et de la Technologie fait cette mise au point pour les allocations de recherche et pour les contrats à durée déterminée des établissements publics appelés «bourses », dans une note du 21 mars 2000 (DAJ- B 1 - MLC/n582, en ligne http://dr.education.fr/Alloc_doc/Statut_allocataires_rech.pdf). recherche. Ainsi, si la plupart des acteurs savent que les doctorants se forment à la recherche, rares sont ceux qui reconnaissent que la thèse est également une activité de travail. Nous avons enquêté sur des doctorants salariés pour leur thèse afin d'analyser comment se définit leur statut socioprofessionnel dans un contexte précis, celui de leur activité dans les laboratoires de recherche.

Le statut des doctorants dans les laboratoires est variable. Des travaux antérieurs suggèrent d'en distinguer trois composantes :

La première est la place des doctorants dans les collectifs de recherche du laboratoire et leur rôle dans le groupe professionnel. Les sciences sociales mettent en évidence deux fonctions à l'activité des doctorants : leur formation assure la reproduction de la communauté scientifique et leur activité est indispensable à la production collective de connaissances (Shinn, 1988; Mangematin 2001). En pratique, quelle fonction prime pour les doctorants et pour les scientifiques titulaires?

\section{Encadré 1}

\section{Les effectifs de la recherche publique en France en 2001-2002}

\begin{tabular}{|l|c|c|}
\hline & Chercheurs & Personnels techniques et administratifs \\
\hline Personnel titulaire & 86000 & 85000 \\
\hline Personnel contractuel dont : & 85000 & - \\
\hline $\begin{array}{l}\text { Enseignement supérieur } \\
\text { lattachés temporaires d'enseignement } \\
\text { et de recherche, moniteurs) }\end{array}$ & 12000 & - \\
\hline Chercheurs non titulaires & 6000 & - \\
\hline Post-doctorants & 3000 & - \\
\hline Doctorants (allocataires ou boursiers) & 20000 & - \\
\hline Doctorants non boursiers & 44000 & 85000 \\
\hline Total général & 171000 & \\
\hline
\end{tabular}

\section{Lecture :}

- Les 85000 personnels techniques et administratifs sont fonctionnaires ou bien employés sous contrat à durée indéterminée. Ils effectuent des tâches scientifiques et techniques sous la responsabilité des chercheurs ou bien se chargent de la gestion administrative des projets. Ces personnels jouent un rôle essentiel dans les sciences expérimentales.

- Les 171000 chercheurs sont titulaires $(60 \%)$ ou contractuels $(40 \%)$.

Les chercheurs titulaires sont fonctionnaires ou bien en contrat à durée indéterminée. Ils sont employés par des établissements d'enseignement supérieur ou bien par des établissements de recherche et technologie.

Les chercheurs contractuels titulaires d'un doctorat sont minoritaires. Certains font exclusivement de la recherche, d'autres enseignent également. Pour simplifier, nous désignons ici par « post-doctorants » tous les chercheurs contractuels titulaires d'un doctorat. Les doctorants forment le groupe le plus important. Si un tiers d'entre eux sont salariés dans le cadre de leur thèse, la majorité ne bénéficie pas de bourse, notamment en sciences humaines et sociales.

Source : Ministère chargé de l'Éducation ; Observatoire de sciences, et des techniques ; Dispositif de production coopérative d'indicateurs inter-institutionnels, in Léridon, 2004. 
Tableau 1

Les principaux financements spécifiques en thèse

\begin{tabular}{|c|c|c|c|c|c|}
\hline \multirow[t]{2}{*}{ Financement } & \multicolumn{2}{|c|}{$\begin{array}{l}\text { Pourcentage dans les } \\
\text { thèses financées (pour les } \\
\text { thèses soutenues en 1999) }\end{array}$} & \multirow[t]{2}{*}{ Type de contrat } & \multirow[t]{2}{*}{ Régime social } & \multirow[t]{2}{*}{$\begin{array}{c}\text { Montant mensuel } \\
\text { brut en } \\
\text { euros (2005) }\end{array}$} \\
\hline & $\begin{array}{c}\text { Chimie et } \\
\text { sciences des } \\
\text { matériaux }\end{array}$ & $\begin{array}{l}\text { Biologie, } \\
\text { médecine, } \\
\text { santé }\end{array}$ & & & \\
\hline $\begin{array}{l}\text { Allocation de recherche du } \\
\text { ministère de l'Éducation } \\
\text { nationale, de l'Enseignement } \\
\text { supérieur et de la Recherche. }\end{array}$ & 37 & 42 & $\begin{array}{l}\text { Contrat à durée } \\
\text { déterminée avec } \\
\text { l'État }\end{array}$ & $\begin{array}{l}\text { Couverture sociale } \\
\text { de droit commun }\end{array}$ & 1410 \\
\hline $\begin{array}{l}\text { Bourses des } \\
\text { établissements de } \\
\text { recherche }\end{array}$ & 13 & 9 & $\begin{array}{l}\text { Contrat à durée } \\
\text { déterminée avec } \\
\text { l'établissement }\end{array}$ & & $\begin{array}{l}\text { De } 1410 \text { à } \\
2000 \text { environ }\end{array}$ \\
\hline $\operatorname{CIFRE}(*)$ & 6 & 3 & $\begin{array}{c}\text { Contrat de travail } \\
\text { à durée déterminée } \\
\text { avec l'entreprise }\end{array}$ & & $\begin{array}{c}\text { Minimum : } 1685 . \\
\text { Salaire moyen : } \\
1970\end{array}$ \\
\hline $\begin{array}{l}\text { Autres (entreprises, } \\
\text { collectivités publiques, } \\
\text { associations...) }\end{array}$ & 36 & 37 & $\begin{array}{c}\text { Variable : contrat } \\
\text { à durée déterminée, } \\
\text { libéralités... }\end{array}$ & $\begin{array}{l}\text { Non unifié }\left({ }^{* *}\right) \text { : } \\
\text { sécurité sociale } \\
\text { étudiante (si moins de } \\
28 \text { ans) ou salariée. }\end{array}$ & $\begin{array}{c}\text { Variable } \\
\text { (non précisé) }\end{array}$ \\
\hline $\begin{array}{l}\text { Financements pour } \\
\text { étranger }(* * *)\end{array}$ & 8 & 9 & Variable & Non unifié & $\begin{array}{c}\text { Variable } \\
\text { (non précisé) }\end{array}$ \\
\hline Total & 100 & 100 & & & \\
\hline
\end{tabular}

(*) Convention industrielle de formation par la recherche. La CIFRE est le principal dispositif de financement des thèses industrielles géré par l'Agence nationale de la recherche technologique (ANRT). Une entreprise embauche un doctorant pendant trois ans, avec une aide de l'ANRT d'environ $15000 €$ par an. L'entreprise verse au laboratoire une somme négociable destinée à couvrir les frais liés à la thèse. Le sujet de thèse répond à la fois à sa demande et à une problématique du laboratoire.

(**) La plus grande association de doctorants et de post-doctorants, la Confédération des jeunes chercheurs, estime que $15 \%$ des doctorants travaillent dans des conditions illégales dans les laboratoires français (CJC, 2004). Leur employeur ne déclare pas leur activité professionnelle pour ne pas payer les charges afférentes.

$(* * *)$ Ces financements sont attribués par le pays d'origine, par le ministère français des Affaires étrangères, par des programmes français et internationaux de développement des échanges scientifiques.

Source : DGRT (2001) et consultation de guides de financement de thèses.

La deuxième composante du statut est leur positionnement dans des relations de travail avec leurs encadrants (Becker et Carper, 1956). Des deux facettes de la relation d'apprentissage entre doctorants et encadrants, relation pédagogique versus relation hiérarchique (Jalaudin et Moreau, 2002), laquelle prédomine et oriente le statut des doctorants?

La dernière composante est leur implication dans les négociations et les conflits du collectif de travail. La sociologie du travail et des relations professionnelles (Reynaud, 1982) avance que cette participation structure les expériences professionnelles. L'attitude des doctorants les situe-t-elle plutôt du côté des étudiants, par leur retrait des débats engagés par les scientifiques titulaires? Les positionne-t-elle inversement du côté des travailleurs, par leur implication dans les discussions et les conflits, comme des membres à part entière du collectif de travail ?

Une enquête ethnographique dans deux laboratoires de biologie et de chimie, Bleu et Vert, nous a permis d'explorer comment se définit le statut socioprofessionnel des doctorants dans ces trois registres. Pourquoi le statut d'étudiant peut-il prédominer dans une situation doublement favorable à l'attribution d'un statut de travailleur? Du fait de consignes strictes de leur université de rattachement, tous les doctorants des laboratoires Bleu et Vert sont en effet salariés pour leur thèse. Par ailleurs, les doctorants de chimie et de biologie sont membres à temps plein d'un collectif de travail en laboratoire. Les conclusions tirées de cette recherche ne 


\section{Encadré 2}

\section{Une enquête ethnographique sur les situations de travail en laboratoire}

\begin{tabular}{|l|l|l|}
\hline \multicolumn{1}{|c|}{ Laboratoire } & \multicolumn{1}{|c|}{ Laboratoire Bleu } & \multicolumn{1}{c|}{ Laboratoire Vert } \\
\hline Période d'enquête & $\begin{array}{l}\text { Présence à temps plein : septembre 2002 } \\
\text { à février 2003. }\end{array}$ & Présence à temps plein : mars à juin 2003. \\
\hline $\begin{array}{l}\text { Nature du travail } \\
\text { ethnographique }\end{array}$ & $\begin{array}{l}\text { - Étude documentaire sur l'évolution du laboratoire. } \\
- \text { Observation du travail, présence à des réunions et au conseil de laboratoire, aux évènements } \\
\text { marquants (soutenances de thèses ou réunions festives, visite d'un comité extérieur d'évaluation), } \\
\text { participation à la vie collective des jeunes chercheurs sur leur lieu de travail et en dehors de } \\
\text { celui-ci. } \\
\text { - Entretiens semi-directifs d'une à deux heures, avec trois volets principaux : un récit biogra- } \\
\text { phique sur le parcours personnel et professionnel de l'interviewé ; une description de ses acti- } \\
\text { vités de travail ; I'organisation et l'emploi dans le laboratoire. }\end{array}$ \\
\hline $\begin{array}{l}\text { Entretiens } \\
\text { semi-directifs }\end{array}$ & $\begin{array}{l}7 \text { personnels techniques et administratifs ; } \\
31 \text { scientifiques titulaires. }\end{array}$ & $\begin{array}{l}\text { - } 11 \text { doctorants et post-doctorants ; } \\
19 \text { scientifiques titulaires. }\end{array}$ \\
\hline
\end{tabular}

peuvent alors probablement pas être transposées aux doctorants en sciences humaines, moins fréquemment financés et/ou intégrés dans un laboratoire. Cette étude offre alors un éclairage localisé sur la question plus générale des processus de reconnaissance d'une activité professionnelle sur le lieu de travail.

Nous exposons tout d'abord les deux fonctions de l'activité des doctorants dans un laboratoire (l'acquisition de compétences en recherche, la production de connaissances scientifiques et technologiques) et nous montrons que ces deux fonctions ont une importance égale dans les laboratoires. Nous suggérons ensuite que les relations de travail avec les encadrants sont déterminantes dans l'attribution d'un statut professionnel et qu'elles divisent les doctorants en deux groupes : les doctorants salariés du public (financés par une allocation ou par une bourse publique), les doctorants salariés d'une entreprise. Nous avançons enfin que le positionnement de l'ensemble des doctorants dans la vie collective du laboratoire les situe du côté des étudiants.

\section{LES DOCTORANTS, PIERRE ANGULAIRE DU TRAVAIL SCIENTIFIQUE}

Dans les sciences expérimentales, la reconnaissance des deux fonctions des doctorants fait l'objet d'un large consensus parmi les scientifiques titulaires et parmi les économistes des sciences : "Ils sont à la fois la principale force de travail des laboratoires et les futurs responsables scientifiques. » (Freeman, R., Weinstein, E., Marincola, et alii. 2001, p. 40). L'emploi de doctorants permet de former à la recherche des jeunes qui sont les futurs scientifiques titulaires du public et du privé. Le travail des doctorants est aussi indispensable pour produire des connaissances scientifiques et technologiques.

Nous montrons d'abord que le laboratoire est, dans ces disciplines, le lieu incontournable de transmission de savoirs de recherche complexes et peu formalisables. Nous expliquons ensuite que les doctorants n'acquièrent pas seulement des compétences, mais qu'ils produisent aussi des connaissances scientifiques avec leur encadrant.

\section{Une formation pratique à la recherche...}

Pour accéder à un emploi de scientifique titulaire dans la recherche publique ou privée, le doctorat est le plus souvent requis. Son obtention sanctionne l'acquisition d'un ensemble de savoirs théoriques et professionnels jugés nécessaires à l'exercice de la recherche. Les savoirs théoriques sont pour l'essentiel transmis lors des cinq années d'études universitaires qui précèdent l'entrée en thèse. 
Parvenus en thèse, les étudiants n'ont plus d'enseignements théoriques. La formation est centrée sur l'acquisition en laboratoire de savoirs professionnels, autrement dit de l'ensemble des compétences grâce auxquelles un chercheur définit, réalise et valorise un projet de recherche. Ces savoirs ont deux caractéristiques: ils concernent principalement la pratique expérimentale et ils supposent une relation pédagogique personnalisée avec l'encadrant.

Tout d'abord, devenir chercheur en chimie ou en biologie passe par une formation pratique au travail expérimental. Ces tâches nommées les «manips» (l'expérimentation requérant de manipuler des produits, des matériaux, des instruments) ou la «paillasse» (le plan de travail pour l'expérimentation en laboratoire) constituent le cœur de métier d'un jeune chercheur. Les doctorants consacrent l'essentiel de leur thèse $\mathrm{e}^{5}$ à apprendre sur le tas à «maniper », acquérant ainsi des savoir-faire peu formalisables. Les doctorants salariés d'une entreprise apprennent aussi à résoudre les problèmes technologiques que pose la mise au point d'un outil ou d'un procédé :

«Ce qui marche à la paillasse en laboratoire ne marche pas toujours en entreprise. Par exemple, on ne peut pas chauffer à des températures aussi élevées ou bien on ne peut pas utiliser les mêmes produits parce que leur toxicité ne peut pas être contrôlée dans les mêmes conditions qu'en laboratoire. » (Doctorant, laboratoire Bleu, CIFRE, troisième année de thèse)

À mesure qu'ils avancent dans leur thèse, les doctorants s'initient aux autres aspects du métier de chercheur: présentation des résultats et rédaction des publications, encadrement de stagiaires, gestion d'un projet (depuis les aspects pratiques comme la commande de matériel, jusqu'aux activités plus complexes comme la réponse aux appels d'offre).

En 2000, la création des écoles doctorales ${ }^{6}$ a introduit une dimension formalisée dans la formation doctorale. Désormais, tous les doctorants suivent une centaine d'heures de formation sur trois ans, dans

\footnotetext{
${ }^{5}$ Soit environ deux ans et demi sur les trois ans que dure en moyenne leur thèse, les six derniers mois étant consacrés à la rédaction du mémoire.

${ }^{6}$ Les écoles doctorales fédèrent, souvent sur une base disciplinaire, des laboratoires qui accueillent des doctorants. Elles attribuent les allocations de recherche du ministère et organisent des formations complémentaires au travail sur le sujet de thèse.
}

leurs domaines scientifiques ou sur des sujets favorisant leur insertion professionnelle ${ }^{7}$. Cette formation élargit les compétences des docteurs. Du fait de son faible volume horaire, elle occupe toutefois une place réduite par rapport à la pratique en laboratoire.

L'acquisition de compétences en recherche suppose ensuite une relation pédagogique personnalisée avec un ou plusieurs encadrants (le directeur de thèse et l'encadrant industriel pour les doctorants salariés d'une entreprise).

La formation par la pratique induit un encadrement étroit du travail expérimental. L'étroitesse de l'encadrement et la forte responsabilité des encadrants visà-vis des doctorants s'expliquent par la nécessité de montrer les gestes techniques, mais aussi par le coût et la dangerosité des expériences. En cours de thèse, à mesure que le doctorant maitrise les manipulations techniques, l'objet de l'encadrement se déplace vers l'analyse et la communication des résultats :

«Au début, on leur montre les techniques et on fait éventuellement une fois les manips avec eux. Ensuite, il faut que les étudiants se lancent, qu'ils refassent les manips seuls, qu'ils réfléchissent à partir des résultats aux conclusions ou bien aux nouvelles manips. Les interactions avec eux se font plus sur les résultats et sur les idées de manip et moins sur des techniques. Au début, on rédige aussi les publis, parce que l'étudiant ne sait pas faire. On lui apprend aussi cela. » (Encadrant, laboratoire Vert: chercheur, douze ans d'ancienneté ; a encadré six doctorants)

La capacité des doctorants à se passer des consignes techniques quasi-quotidiennes, la possibilité pour les encadrants d'espacer les réunions de travail (rythme hebdomadaire ou bimensuel), le rythme d'avancement du travail (les résultats expérimentaux obtenus, la qualité des analyses...) permettent aux encadrants d'évaluer si les doctorants acquièrent des savoirs professionnels.

\section{... qui fournit les résultats expérimentaux}

Pour les laboratoires, la thèse n'a pas pour seule fonction de transmettre des savoirs professionnels. Les

\footnotetext{
${ }^{7}$ Les « Doctoriales» en font partie. Ces stages de construction d'un projet personnel et professionnel sont suivis généralement en deuxième année de thèse.
} 
difficultés des docteurs à trouver un emploi stable dans la recherche publique ou privée et les reconversions de certains vers d'autres secteurs d'activité font en effet peser une forte incertitude sur la rétribution du temps et des crédits engagés dans cette formation.

Les scientifiques titulaires trouvent aussi un intérêt immédiat à former des doctorants car le travail expérimental de ces derniers fournit la matière première, à savoir les résultats expérimentaux, à la plupart de leurs projets. Les biologistes comme les chimistes travaillent collectivement, dans une équipe d'environ cinq personnes. Un ou deux scientifiques titulaires définissent le projet et le pilotent, en collaboration avec des industriels pour les thèses avec une entreprise.

Dans le laboratoire, le(s) porteur(s) du projet et les autres titulaires (chercheurs, parfois ingénieurs ou techniciens) effectuent une partie du travail expérimental, mais les doctorants sont les principaux artisans des «manips ». Ils s'y consacrent en effet à temps plein, là où l'emploi du temps des scientifiques titulaires se répartit sur de multiples activités (encadrement, enseignement, administration...).

Les scientifiques titulaires conditionnent ainsi le lancement d'un projet à la possibilité d'y affecter un étudiant de master, un doctorant et plus rarement un post-doctorant ${ }^{8}$. L'emploi de doctorants salariés d'une entreprise est également essentiel pour démarrer de longues collaborations industrielles. Encadrer des étudiants de master puis des doctorants permet plus généralement de faire avancer les recherches de l'équipe :

"Quand je n'étais pas là parce que j'étais en enseignement par exemple, mon projet s'arrêtait, éventuellement mes cultures aussi étaient foutues. C'était un éternel recommencement et rien ne sortait de ces recherches. Je me suis arrangée pour mettre mes enseignements au premier semestre, de façon à avoir un stagiaire de DEA (diplôme d'étude approfondi) au second semestre pour faire avancer la recherche. On a eu de la chance parce qu'il a eu une bourse de thèse derrière." (Encadrante, laboratoire Bleu: ensei-

\footnotetext{
${ }^{8}$ En France, les possibilités d'emploi de post-doctorants se développent mais elles restent réduites en comparaison d'autres pays. Plusieurs rapports publics, notamment le rapport Fréville (2001), l'expliquent par la crainte des gouvernements successifs de créer des viviers de chercheurs contractuels.
}

gnant-chercheur, six ans d'ancienneté; a encadré deux doctorants).

L'encadrement de thèses apporte également une reconnaissance scientifique et institutionnelle aux scientifiques titulaires. Selon les conventions en vigueur en chimie et en biologie, ils signent en dernière position les publications issues des travaux qu'ils encadrent, qui sont principalement des travaux de thèse ${ }^{9}$. Le nombre de publications comme « dernier auteur » constitue un critère essentiel pour progresser dans une carrière publique en recherche dans les sciences expérimentales. Les brevets, que le laboratoire et l'entreprise déposent parfois suite à un travail de thèse en commun, prennent de l'importance dans l'évaluation de l'activité des chercheurs du public.

Les institutions de tutelle des laboratoires savent aussi que les doctorants sont au cœur de l'activité de recherche. Elles incluent les effectifs et les financements des doctorants dans leurs indicateurs d'évaluation. La diversité des financements de thèse (cf. encadré 3) signale notamment que les scientifiques titulaires sont bien reconnus ou bien insérés dans des réseaux extérieurs.

Enfin, les doctorants soulagent ponctuellement leur équipe de tâches incontournables. Ils prêtent main forte à d'autres expérimentations, encadrent des stagiaires de licence et de master, forment des arrivants (étudiants et chercheurs) à l'utilisation de certains appareils. Ce travail est d'autant plus utile que le personnel technique ${ }^{10}$ est parfois insuffisant.

Ainsi, les laboratoires reconnaissent que les doctorants sont les forces vives de leur recherche. Cette reconnaissance rejoint plusieurs travaux sur la place des doctorants dans la division du travail et sur la spécificité des savoirs qu'ils produisent. T. Shinn (1988) montre que les doctorants produisent des résultats locaux, sont sensibles à la complexité de l'analyse, détectent les anomalies et perfectionnent les méthodes. Les chercheurs seniors utilisent ces résultats locaux pour réajuster leurs modèles.

\footnotetext{
${ }^{9}$ Les doctorants signent ces mêmes publications en première position, réservée aux contributeurs principaux à l'expérimentation. ${ }^{10}$ Les ingénieurs et les techniciens sont affectés à une équipe ou bien à des services d'instrumentation communs au laboratoire. Le personnel technique affecté à une équipe n'est pas assez nombreux pour se charger de toutes les tâches collectives mentionnées, notamment en période d'accueil des stagiaires (au printemps).
} 
V. Mangematin (2001) avance que les doctorants maîtrisent des techniques de pointe et possèdent des savoir-faire précis et fortement contextualisés, qui se transmettent imparfaitement par les publications. Mobiles après leur thèse ${ }^{11}$, les doctorants jouent alors un rôle essentiel dans la circulation de ces savoir-faire à l'intérieur et à l'extérieur du monde académique.

$\mathrm{Au}$ total, les acteurs des laboratoires attribuent un poids égal aux deux facettes de l'activité des doctorants : d'un côté, l'acquisition de compétences en recherche; de l'autre, la contribution à la production scientifique.

L'activité des doctorants, comme celle des apprentis d'autres professions qualifiées, par exemple les huissiers stagiaires (Mathieu-Fritz, 2005), se situe sur un continuum entre deux pôles. Certains constituent plutôt une main-d'œuvre bon marché. Pour les doctorants financés par une entreprise, cette position se traduit par l'exécution de missions d'ingénierie ou de production au détriment du travail de recherche. Pour les doctorants salariés du public, cette place comme main-d'œuvre bon marché conduit à l'exécution de tâches lourdes, peu gratifiantes ou extérieures à leur thèse (l'encadrement de stagiaires de licence, expériences sur d'autres projets). À l'opposé, d'autres doctorants sont principalement considérés comme de futurs professionnels. Ils se concentrent sur les activités les plus formatrices ou les plus rentables pour leur dossier scientifique. L'investissement dans la relation pédagogique peut alors être plus lourd, mais il a un double bénéfice pour l'encadrant et pour le système de recherche : l'un immédiat et certain (la qualité de la production scientifique issue de la thèse), l'autre différé et aléatoire (l'intégration pérenne d'un bon chercheur à la communauté scientifique).

L'analyse de l'activité des doctorants ne permet ainsi pas de déduire que leur statut dans les laboratoires se rapproche davantage de celui d'un étudiant ou de celui d'un travailleur à part entière. La sociologie interactionniste des professions (Becker et Carper, 1956) montre néanmoins que le statut socioprofessionnel ne dérive pas mécaniquement du contenu de l'activité et de la fonction occupée. Le statut se définit aussi dans des engagements subjectifs au travail qui résultent d'une part de la trajectoire anté-

${ }^{11}$ et pendant la thèse, pour les doctorants salariés d'une entreprise. rieure et des anticipations professionnelles, d'autre part des relations de travail (des attentes réciproques et des attributions de rôle). En analysant le statut socioprofessionnel en thèse de ce point de vue, deux catégories de doctorants se dégagent clairement : les salariés d'une entreprise et les salariés du public.

\section{DES POSITIONNEMENTS DIVERGENTS VIS-À-VIS DES ENCADRANTS, DE LA FORMATION ET DU TRAVAIL}

Nous analysons tout d'abord la divergence des projets et des parcours professionnels des doctorants salariés d'une entreprise et du public. Nous montrons ensuite que la poursuite de ces projets instaure des relations différentes entre encadrants et doctorants. L'inscription dans des relations pédagogiques et/ou hiérarchiques explique le poids que chaque catégorie attribue à sa formation et à son travail.

\section{La divergence des projets et des parcours}

Les résultats de notre enquête ( $c f$. encadré 3 ) confirment plusieurs études sur les doctorants et les docteurs en France (notamment De Lassalle, Maillard, Martinelli et al. (1999) et Mangematin et Robin (1999)). Comme les docteurs de leur discipline (cf. tableau 2), ceux des laboratoires Bleu et Vert obtiennent difficilement un emploi stable. Trois ans après leur soutenance, les docteurs en chimie et en biologie sont ainsi davantage au chômage ou en contrat à durée déterminée que d'autres docteurs en sciences exactes (Giret, 2005). La majorité qui choisit la recherche publique est employée pendant plusieurs années en contrat à durée déterminée en France ou à l'étranger. Beaucoup ne trouvent ensuite pas d'emploi titulaire et se reconvertissent tardivement dans un emploi privé (Giret, 2005).

Au-delà d'une difficulté commune à réaliser leur projet, l'orientation professionnelle que privilégient les doctorants se différencie fortement selon qu'ils sont salariés du privé ou du public.

Seuls les doctorants salariés d'une entreprise déclarent qu'ils occuperont par la suite un emploi privé. L'accès à la recherche publique de leurs prédéces- 
Tableau 2

Devenir professionnel en 2004 des docteurs en sciences de 2001

\begin{tabular}{|l|c|c|c|c|c|}
\hline & $\begin{array}{c}\text { Chercheurs dans } \\
\text { le public }\end{array}$ & $\begin{array}{c}\text { Autres professions } \\
\text { du public }\end{array}$ & Emploi privé & Total & $\begin{array}{c}\text { dont emplois à } \\
\text { durée limitée } \\
\text { (secteur public et privé) }\end{array}$ \\
\hline Chimie & $43 \%$ & $9 \%$ & $48 \%$ & $100 \%$ & $30 \%$ \\
\hline $\begin{array}{l}\text { Sciences de la vie } \\
\text { et de la terre }\end{array}$ & $45 \%$ & $16 \%$ & $39 \%$ & $100 \%$ & $32 \%$ \\
\hline $\begin{array}{l}\text { Ensemble des } \\
\text { disciplines }\end{array}$ & $47 \%$ & $15 \%$ & $38 \%$ & $100 \%$ & $24 \%$ \\
\hline
\end{tabular}

Source : Céreq, in Giret (2005).

seurs a en effet été faible, voire nul, sur les quinze dernières années ( $c f$. encadré $3: 7 \%$ à Bleu, $0 \%$ à Vert). La plupart des doctorants salariés du public interrogés souhaitent à l'inverse rester dans la recherche publique et ne réfléchissent pas encore à d'autres options professionnelles. Cette orientation vers les emplois académiques est préoccupante pour ces doctorants. Elle signifie qu'ils ne préparent pas une réorientation vers des emplois hors recherche qui sera pourtant inéluctable pour un bon nombre d'entre eux, compte tenu du taux de réussite du projet académique des doctorants salariés du public dans les deux laboratoires ( $c f$. encadré 3 : $33 \%$ à Bleu, $62 \%$ à Vert).

Cette opposition entre les projets et les parcours professionnels confirme l'analyse de Becker et Carper des mécanismes de l'identification professionnelle des étudiants en master et des doctorants (Becker, Carper, 1956, op. cit.). L'identification des doctorants à des postes, le fait de les estimer accessibles et attrayants, leurs engagements privilégiés envers certaines tâches («commitment to task») dépendent des contacts noués pendant la thèse, des systèmes informels de parrainage, de recommandation et de contrôle. Ainsi, l'absence d'intérêt, voire le rejet des emplois privés par les doctorants salariés du public s'explique en grande partie par leur méconnaissance de ces emplois. Inversement, l'intérêt des doctorants salariés d'une entreprise pour la recherche en entreprise se renforce à mesure qu'ils travaillent sur un projet industriel et qu'ils repèrent plusieurs possibilités d'emploi en entreprise.

Pour préparer la suite de leur parcours professionnel, les doctorants salariés d'une entreprise et du public n'entretiennent pas les mêmes relations avec leurs encadrants. Dans l'entreprise, l'encadrement des doctorants salariés du privé relève de relations hiérarchiques, axées sur l'apport de résultats à l'employeur. Dans le laboratoire, l'encadrement de tous les doctorants par un scientifique titulaire relève plutôt de relations pédagogiques, centrées sur l'acquisition de compétences en recherche.

\section{Les doctorants salariés d'une entreprise : de jeunes collègues}

Du fait des champs disciplinaires respectifs des laboratoires Bleu et Vert, les doctorants salariés d'une entreprise sont plus nombreux dans le laboratoire Bleu ( $c f$. encadré 3). Ils ont été par le passé employés sous plusieurs types de contrats ; toutefois, ceux que nous avons interviewés étaient tous employés en CIFRE.

Les doctorants CIFRE travaillent sous la double direction d'un employeur privé et d'un encadrant dans le laboratoire. De manière schématique, les attentes de l'employeur privé concernent plutôt la production du doctorant, l'apport de solutions et la résolution de problèmes, tandis que celles de l'encadrant dans le laboratoire relèvent davantage de sa formation à la recherche et de l'avancée des connaissances fondamentales :

"Quand un industriel s'engage dans une thèse, il a des attentes. Il veut qu'un projet avance concrètement, même si au bout de la thèse on n'arrive pas complètement à synthétiser la molécule avec les propriétés voulues. En même temps, l'objectif d'une thèse pour nous c'est aussi que l'étudiant approfondisse son domaine de compétences. Ça peut poser 
problème si on veut passer plus de temps sur une voie de synthèse intéressante. 》 (Encadrant, laboratoire Bleu : chercheur, trente ans d'ancienneté ; a encadré environ quinze thèses industrielles)
La conciliation ou l'opposition entre ces objectifs, leur traduction dans la supervision concrète du travail, enfin les attentes et les jugements à l'encontre du doctorant, varient selon le lieu de travail du docto-

\section{Encadré 3}

\section{Présentation synthétique des laboratoires enquêtés}

\begin{tabular}{|c|c|c|}
\hline & Laboratoire Bleu & Laboratoire Vert \\
\hline Création & 1966 & 1974 \\
\hline Rattachements institutionnels & $\begin{array}{c}\text { Unité propre du CNRS } \\
\text { (département des sciences } \\
\text { chimiques), associée à l'université. }\end{array}$ & $\begin{array}{l}\text { Unité mixte CNRS (département } \\
\text { des sciences du vivant), CEA, } \\
\text { INRA (*), université. }\end{array}$ \\
\hline Activités principales & $\begin{array}{l}\text { Chimie et biologie, sur des } \\
\text { molécules végétales. }\end{array}$ & Biologie végétale. \\
\hline Effectifs en 2003 & $\begin{array}{l}\text { - } 26 \text { scientifiques titulaires. } \\
-23 \text { doctorants. } \\
\text { - } 12 \text { post doctorants. } \\
\text { - } 27 \text { personnels techniques } \\
\text { et administratifs. }\end{array}$ & $\begin{array}{l}\text { - } 24 \text { scientifiques titulaires. } \\
-8 \text { doctorants. } \\
-5 \text { post doctorants. } \\
-12 \text { personnels techniques } \\
\text { et administratifs. }\end{array}$ \\
\hline $\begin{array}{l}\text { Nombre de thèses soutenues } \\
\text { depuis } 1992\end{array}$ & 64 & 16 \\
\hline $\begin{array}{l}\text { Répartition des financements des } \\
\text { thèses soutenues depuis } 1992 \\
\text { Doctorants salariés du public } \\
\text { (Allocations de recherche et bourses). } \\
\text { Doctorants salariés d'une entreprise } \\
\text { (CIFRE, contrats industriels). } \\
\text { Financements pour doctorants étrangers } \\
\text { Inconnu } \\
\text { Total }\end{array}$ & $\begin{array}{r}53 \% \\
27 \% \\
8 \% \\
12 \% \\
100 \%\end{array}$ & $\begin{array}{r}94 \% \\
6 \%\end{array}$ \\
\hline $\begin{array}{l}\text { Devenir professionnel de ces docteurs } \\
\text { Scientifiques titulaires dans le secteur }\end{array}$ & $20 \%$ & $58 \%$ \\
\hline $\begin{array}{l}\text { public trançais. } \\
\text { Post-doctorants } \\
\text { Emploi à l'étranger (doctorants français } \\
\text { et étrangers) }\end{array}$ & $\begin{array}{l}3 \% \\
14 \%\end{array}$ & $\begin{array}{r}7 \% \\
21 \%\end{array}$ \\
\hline $\begin{array}{l}\text { Emplois privés et emplois publics } \\
\text { hors recherche. }\end{array}$ & $63 \%$ & $14 \%$ \\
\hline Total & $100 \%$ & $100 \%$ \\
\hline $\begin{array}{l}\text { Part des doctorants salariés du public } \\
\text { parmi les scientifiques titulaires dans } \\
\text { le secteur public français. }\end{array}$ & $93 \%$ & $100 \%$ \\
\hline $\begin{array}{l}\text { Taux d'accès des doctorants salariés du } \\
\text { public aux emplois titulaires dans la } \\
\text { recherche publique française. }\end{array}$ & $33 \%$ & $62 \%$ \\
\hline
\end{tabular}

$(*)$ : CNRS : Centre national de la recherche scientifique ; CEA : Commissariat à l'énergie atomique ; INRA : Institut national de la recherche agronomique. Le CNRS, le CEA et l'INRA sont trois grands établissements publics de recherche et de technologie.

Lecture : parmi les doctorants qui ont soutenu leur thèse au laboratoire Bleu depuis 1992, 53 \% étaient salariés du public, autrement dit étaient rémunérés par une allocation de recherche ministérielle ou par une bourse d'une institution publique. Un tiers de ces doctorants salariés du public (33\%) ont accédé après leur thèse à un emploi titulaire dans la recherche publique française.

Source : archives des laboratoires et enquête auprès d'anciens doctorants. 
rant (laboratoire ou entreprise) et selon les négociations autour du contenu des projets. Nous avons ainsi rencontré deux situations opposées.

Un premier doctorant travaillait à temps plein au laboratoire sous la direction de son encadrant universitaire. L'entreprise fixant peu de consignes, il ressentait une pression plus forte vis-à-vis de l'acquisition de compétences en recherche plutôt que par rapport à l'obtention de résultats opérationnels pour l'entreprise.

Un second doctorant alternait des périodes de plusieurs mois de travail au laboratoire et dans l'entreprise. Les encadrants en entreprise et l'encadrant universitaire cherchaient à imposer leurs objectifs respectifs lorsque le doctorant était dans leurs locaux :

"Chaque réunion séparée avec l'un ou avec l'autre faisait dévier les recherches d'un côté ou de l'autre: "produis vite" ou "il faut d'abord comprendre!"” (Doctorant CIFRE, laboratoire Bleu, fin de troisième année de thèse).

Quelles que soient les exigences associées, travailler pour une entreprise a toujours des conséquences sur le positionnement professionnel des doctorants CIFRE. Ceux-ci se situent en effet dans une relation hiérarchique vis-à-vis de leurs interlocuteurs en entreprise. Ils ont la conviction d'apporter des savoirfaire, une expertise, une solution en échange de leur salaire. Lorsqu'ils travaillent avec leur encadrant en laboratoire, ils se positionnent davantage dans une relation pédagogique : leur encadrant leur apporte les connaissances et les savoir-faire et évalue leur progression dans leur formation à la recherche.

La relation hiérarchique en entreprise leur confère un statut professionnel de «jeune collègue », autrement dit de membre à part entière d'un collectif de travail : «Ils me prennent pour un employé à part entière et pas pour un étudiant, donc ils attendent de moi des résultats. Au début ça s'est très bien passé, ensuite un peu moins parce que j'ai eu moins de résultats. L'encadrant de l'entreprise il comprend, c'est aussi un chercheur, mais il n'est pas là pour compatir. » (Doctorant CIFRE, laboratoire Bleu, troisième année de thèse)

La signature d'un contrat de travail avec un employeur privé, la connaissance des règles et des usages de l'entreprise qui les emploie ${ }^{12}$, confronte

${ }^{12}$ Notamment en matière de temps de travail, de congés, de rémunération (augmentations et primes). enfin les doctorants CIFRE aux questions classiques $\mathrm{du}$ monde salarial et conforte leur position de «jeunes collègues » qui travaillent parfois hors les murs de l'entreprise.

\section{Dans le public : un statut de « futurs collègues » du laboratoire}

Les activités des doctorants salariés du public sont diverses. Certains étudient un sujet entièrement défini par leur encadrant et communiquent exclusivement leurs résultats via des conférences et des publications académiques. D'autres collaborent avec les commanditaires d'un contrat ou avec les partenaires d'un projet. Dans toutes ces configurations, les relations avec le directeur de thèse sont centrales et essentiellement d'ordre pédagogique.

L'évaluation des activités en thèse se structure tout d'abord d'après la dynamique antérieure d'apprentissage de la recherche et d'après les compétences attendues d'un scientifique titulaire (Sait-il définir un protocole de recherche, rédiger une publication en anglais, présenter ses résultats à des collègues, etc. ?). Ce schéma pédagogique fait apparaître certaines tâches comme des entraînements au métier plutôt que comme une activité professionnelle. Les conférences entrainent par exemple les doctorants à l'expression orale et à la sociabilité, tandis que la diffusion de leurs résultats scientifiques passe au second plan.

La relation entre doctorants et encadrants est aussi principalement jugée à l'aune de sa qualité pédagogique. Les doctorants apprécient ou critiquent les différentes étapes de leur formation (voir supra « une formation pratique à la recherche»), ainsi que le temps que leur encadrant passe en démonstration, aide, explication...

Certains titulaires avancent qu'une bonne relation pédagogique fait progressivement passer le doctorant du statut d'étudiant ou de "futur collègue », qui apprend pour intégrer les métiers de la recherche, à celui de « jeune collègue » qui est l'égal des scientifiques titulaires. La soutenance sanctionne pour eux l'acquisition des compétences d'un chercheur. Elle marque aussi son entrée dans des relations de travail collégiales ou hiérarchiques, dont l'objet n'est plus de transmettre des savoirs mais de produire ensemble des résultats : 
"Quand les étudiants arrivent au laboratoire, les relations sont déséquilibrées. Ils sont dans un schéma pèrefils ou maître-élève et il faut les aider à rééquilibrer les choses. » (Encadrant au laboratoire Vert; chercheur, six ans d'ancienneté, encadrement de deux doctorants)

Lintervention d'interlocuteurs extérieurs au laboratoire contrebalance peu le poids des relations pédagogiques avec le directeur de thèse. Les partenaires ou les commanditaires des travaux de thèse attendent du doctorant qu'il produise des résultats (par exemple, synthétiser une molécule ou découvrir sa fonction). Le directeur de thèse reste néanmoins le prescripteur, l'évaluateur et le destinataire principal du travail de thèse.

Les propos suivants de ce doctorant, employé sur un contrat européen, contrastent ainsi avec ceux des doctorants CIFRE. Fournir des résultats à des partenaires extérieurs n'est pas suffisant pour qu'il se définisse comme leur « jeune collègue » :

«On est de plus en plus autonome en cours de thèse mais on est toujours en formation. Alors, avec les autres membres du projet européen, on a des réunions tous les 6 mois et au bout de 24, 30, 36 mois il faut fournir des composés. » (Doctorant, laboratoire Bleu; financement par un contrat européen, deuxième année de thèse)

Au total, les doctorants salariés d'une entreprise (ici des doctorants CIFRE) se positionnent dans des relations pédagogiques en laboratoire et dans des relations hiérarchiques en entreprise. Cela les conduit à se reconnaître plus facilement comme des «jeunes collègues » que les doctorants salariés du public qui se situent exclusivement dans une relation pédagogique avec leur directeur de thèse.

Dans la vie quotidienne du laboratoire, les relations pédagogiques priment néanmoins pour l'ensemble des doctorants. Le statut qui leur est attribué se rapproche alors de celui d'étudiant ou de «futur collègue ». La sociologie des relations professionnelles suggère que leur retrait vis-à-vis des conflits du travail les prive d'expériences structurantes du positionnement comme travailleur (Reynaud, 1982, op. cit.).

\section{AU SEIN DU LABORATOIRE, DES DOCTORANTS EN RETRAIT}

Nous montrons tout d'abord que les scientifiques titulaires impliquent difficilement les doctorants dans la gestion du laboratoire. Nous avançons ensuite que les doctorants se placent eux-mêmes en marge de cette vie collective et nous expliquons cette attitude par la prépondérance des relations pédagogiques au sein du laboratoire.

\section{Incompétents pour les affaires collectives?}

Les personnels titulaires participent aux discussions ou aux décisions relatives aux affaires générales de leur équipe ou du laboratoire (demandes de crédits, de postes et de bourses, achats d'équipement, candidatures aux emplois titulaires, partenariats de recherche, positionnement vis-à-vis des institutions de tutelle...). En revanche, les doctorants ne sont pas spontanément inclus parmi les personnes compétentes pour des problèmes administratifs ou gestionnaires.

Dans leur équipe, ils donnent parfois leur avis sur l'achat du petit matériel destiné à leur domaine expérimental, mais les discussions stratégiques se déroulent souvent en leur absence :

«Les problèmes de gestion du groupe ne concernant pas les non-permanents sont traités en comité restreint, comme la réunion du XX où K. a pris la direction et où nous avons traité toutes les questions en cours, comme les collaborations, les arrivées de stagiaires, le comité d'évaluation, l'arrivée d'un chercheur, le fonctionnement du groupe. » (Ingénieur de recherche, laboratoire Bleu : trente ans d'ancienneté dans le laboratoire)

À l'échelle du laboratoire, les scientifiques titulaires font peu appel à leur expertise sur un sujet, une technique ou un enseignement et ils se réservent les questions porteuses d'enjeux importants. Les membres du conseil de laboratoire ${ }^{13}$ sollicitent ainsi rarement le représentant élu des doctorants pour qu'il donne son avis ou apporte des informations.

Associant étroitement la thèse à un temps de formation, les scientifiques titulaires intègrent en effet difficilement cette période dans l'ancienneté profes-

\footnotetext{
${ }^{13}$ Le conseil de laboratoire est une instance consultative composée de représentants nommés et élus du personnel. Le directeur du laboratoire le consulte sur le fonctionnement et l'évolution du laboratoire (politique scientifique, gestion du personnel et des budgets...).
} 
sionnelle dans la recherche. L'extrait suivant du conseil de laboratoire à Bleu montre que certains titulaires ne pensent pas que les années de thèse d'un jeune docteur lui donnent une compétence de gestion dans son domaine.

Son directeur cherche un scientifique titulaire pour représenter Bleu auprès d'une institution locale. Il suggère alors le nom de Serge, ancien doctorant récemment recruté comme chercheur. Face à la réticence de certains membres du conseil de laboratoire, un chercheur rejette l'argument « du nouveau venu » :

« Je voudrais prendre la défense de Serge. Tu dis qu'il est jeune, qu'il est ici depuis trois mois, mais je te rappelle quand même qu'il est ici depuis cinq ans. » (Encadrant, laboratoire Bleu : chercheur, quinze ans d'ancienneté, a encadré douze thèses environ)

\section{Une participation réduite aux échanges collectifs}

Les doctorants contribuent aussi à renforcer leur retrait par rapport aux débats. Assistant à des conseils de laboratoire, nous avons constaté que les autres représentants donnent leur avis, s'opposent, demandent des informations, expriment leur incompréhension, leur étonnement ou leur ignorance. Le représentant des doctorants entre très peu dans ces jeux d'interactions gestuelles ou verbales et s'en tient à une écoute passive.

Ainsi, les doctorants adoptent une attitude de «loyauté » plutôt que de "prise de parole» (Hirschman, 1995) dans les échanges collectifs. En d'autres termes, ils donnent peu leur opinion, émettent rarement des critiques ou des propositions sur le fonctionnement du laboratoire ou sur leurs relations aux encadrants, même lorsqu'ils n'en sont pas satisfaits.

La réserve des doctorants vis-à-vis de leurs problèmes s'explique d'abord par leur absence de familiarité avec les problèmes administratifs et politiques. Les scientifiques titulaires centrent l'activité en thèse sur le travail expérimental et beaucoup voient dans l'initiation aux procédures officielles et aux jeux stratégiques une perte de temps. Ayant ainsi rarement l'occasion de développer leurs capacités d'analyse et de jugement dans ces domaines, les doctorants se désintéressent d'échanges peu intelligibles :
«La dernière fois, je me suis vraiment embêté parce que j'ai passé trois heures à écouter les budgets [...]. En fait, c'est vraiment la vie d'un laboratoire, ce n'est pas hors scientifique mais à mon échelle, je vois ça comme quelque chose de très administratif, de très paperasse. » (Représentant des doctorants au laboratoire Bleu : allocataire de recherche, troisième année de thèse)

Ensuite, la prédominance d'une relation pédagogique entre encadrants et doctorants engendre une difficulté à prendre la parole sur des questions qui ne relèvent pas de la pédagogie. Les récriminations courantes dans une relation hiérarchique (charge de travail, prise des congés, conditions de travail...) restent le plus souvent de l'ordre de l'aparté. Les doctorants les jugent secondaires par rapport à une relation pédagogique qu'ils ne veulent pas compromettre par un conflit. Les seuls problèmes que les doctorants s'autorisent à soulever ouvertement devant leur encadrant, dans le laboratoire ou devant les évaluateurs extérieurs, relèvent de la qualité de leur formation ou des débouchés professionnels qu'elle leur ouvre.

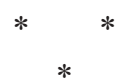

Les appels à la reconnaissance des doctorants comme travailleurs à part entière se sont multipliés en France, notamment sous l'effet de la montée en puissance d'associations de jeunes chercheurs et des débats suscités par les mouvements des chercheurs en 2004.

Notre enquête suggère que les doctorants en sciences expérimentales n'ont pas un statut professionnel unifié dans les laboratoires. Leur activité les positionne autant comme des étudiants que comme des travailleurs. En effet, les laboratoires reconnaissent qu'ils leur transmettent des savoirs professionnels (savoirs-faire techniques; compétences d'encadrement et de diffusion des résultats) et qu'ils utilisent leur production scientifique. En revanche, la relation pédagogique avec leur directeur de thèse situe tous les doctorants comme de "futurs collègues » dans le laboratoire plutôt que comme des travailleurs. Seuls les salariés d'une entreprise se définissent aussi comme des travailleurs opérationnels ou des « jeunes collègues » vis-à-vis de l'entreprise car ils y sont engagés dans une relation hiérarchique. La réserve de tous les doctorants vis-à-vis de la vie collective du laboratoire les cantonne enfin dans un statut d'étu- 
diant, où ils sont peu autorisés et où ils s'autorisent peu à prendre position dans les débats des scientifiques titulaires.

Ces conclusions invitent à proposer qu'un tiers, n'entretenant pas de relation pédagogique avec le doctorant, suive le déroulement de chaque thèse. Un rapport sur le doctorat en France (FutuRIS, 2005, op. cit.) propose que l'École doctorale joue ce rôle et passe un contrat individualisé avec chaque doctorant. Ce contrat, en clarifiant le contenu et les objectifs de chaque thèse, afficherait le doctorat comme une expérience professionnelle et aiderait les acteurs de la thèse (doctorant, directeur de thèse, laboratoire, École doctorale) à reconnaître que les doctorants sont autant des travailleurs que des étudiants.

Notre recherche suggère ensuite que le système de la recherche bénéficierait de la participation des doctorants à sa gestion (à l'échelle des laboratoires ou des institutions qui les emploient) car ils possèdent une riche expérience sur des problèmes centraux de la recherche: le travail expérimental, l'articulation entre l'enseignement supérieur et la recherche (via l'encadrement de stages ou les enseignements), enfin les collaborations industrielles (pour les salariés d'une entreprise). Les moteurs français de cette participation et plus généralement de la reconnaissance de leur activité professionnelle sont encore largement à définir, là où ils s'appuient sur un relais syndical spécifique dans d'autres pays ${ }^{14}$.

Cette analyse des ambivalences du statut des doctorants et de sa construction dans les laboratoires suggère enfin que la définition du « travail » (par opposition à la formation, mais aussi au hors travail ou au loisir) n'est pas générique. Dans chaque activité, elle relève de plusieurs processus de qualification et de catégorisation dans lesquels s'engagent les juristes, les acteurs sociaux, enfin les professionnels sur le lieu de travail.

14 Notamment au Québec (Fédération Canadienne des étudiantes-étudiants Québec) et aux États-Unis, voir Freeman, Weinstein, Marincola, et al. (2001) op. cit. Ces auteurs signalent la création de plusieurs syndicats pour obtenir des accords collectifs sur les salaires et sur les conditions de travail des scientifiques contractuels. Ces accords portent pour le moment sur leurs activités d'enseignement, mais ces syndicats cherchent aussi à peser sur les conditions d'exercice de la recherche en thèse et après la thèse.

\section{Bibliographie}

Becker H., Carper J. (1956), «The elements of identification with an occupation», American Sociological Review, vol. 21, n 3, pp. 341-348.

CJC (2004), Rapport sur les conditions de travail illégales des jeunes chercheurs, $8 \mathrm{p}$.

De Lassalle M., Maillard D., Martinelli D., Perret C., Paul J.-J. (1999), De la compétence universitaire à la qualification professionnelle. L'insertion des docteurs, Document de synthèse ${ }^{\circ} 114$, Céreq, $130 \mathrm{p}$.

DGRT (2001), Rapport sur les études doctorales 2000, ministère de la Recherche et de la Technologie.
Freeman R., WeinsteinE., Marincola E., Rosenbaum J., Salomon F. (2001), Careers and Rewards in Bio Sciences : the disconnect between scientific progress and career progression. The American Society for Cell Biology, 53 p.

Fréville Y. (2001), Rapport d'information sur la politique de recrutement et de gestion des universitaires et des chercheurs, Rapport $n^{\circ} 54$ au Sénat.

FutuRIS (2005), Propositions pour favoriser l'emploi des docteurs. Résultat du groupe de travail FutuRIS, Lehmann J.-C. (Président), Fixaris D. et Pallez F. (rapporteurs), $40 \mathrm{p}$. 
Giret J.-F. (2005), « De la thèse à l'emploi. Les débuts professionnels des jeunes titulaires d'un doctorat», Bref-Céreq, 220, 8 p.

Hirschman A. O. (1995), Défection et prise de parole, Théorie et applications, Fayard, coll. «L'espace du politique », Paris, $216 \mathrm{p}$.

Jalaudin C., Moreau G. (2002), «Transmettre le métier: les complexités de la relation maître/apprenti », in Piotet, F. (dir.), La révolution des métiers, Paris, PUF, pp. 53-76.

Léridon H. (2004), «L'avenir de la recherche en France : perspectives démographiques » Population et sociétés, $\mathrm{n}^{\circ} 403$.

Mangematin V., Robin S. (1999), L'insertion professionnelle des docteurs en Sciences de la Vie (1984-
1998) : formation des chercheurs et acquisition des compétences, INRA / SERD, Grenoble.

Mangematin V. (2001), « Les docteurs, producteurs et diffuseurs de connaissances ", in Mustar P., Penan H. (dir.), Encyclopédie de l'innovation, Paris, Économica.

Mathieu-Fritz A. (2005) "L'intégration au groupe professionnel des huissiers de justice », Formation Emploi, 89, pp. 5-18.

Reynaud J.-D. (1982), Sociologie des conflits $d u$ travail, Paris, PUF.

Shinn T. (1988), "Hiérarchies des chercheurs et formes de recherche », Actes de la recherche en sciences sociales, vol. 74, pp. 2-22.

\section{Résumé}

\section{Les doctorants en sciences expérimentales : futurs collègues ou jeunes collègues? Séverine Louvel}

Les doctorants salariés durant leur thèse occupent un double statut : ce sont à la fois des étudiants et des travailleurs. L'exemple de doctorants en thèse de chimie et de biologie permet de pointer la difficile reconnaissance du statut de travailleur sur le lieu d'activité, les laboratoires. L'article analyse la place de la formation et du travail dans trois registres de construction du statut socioprofessionnel en thèse : I'activité, les relations de travail, la participation à la vie collective du laboratoire. Les deux derniers registres positionnent les doctorants davantage comme des "futurs collègues", soit des étudiants qui intègreront ultérieurement les métiers de la recherche, plutôt que comme des "jeunes collègues", membres opérationnels à part entière d'un collectif de travail. Ces analyses sont fondées sur une enquête ethnographique dans deux laboratoires.

\section{Mots clés}

Identité professionnelle, métier de la recherche, thèse, travail étudiant Journal of Economic Literature : I 23, J 24 